\title{
Acknowledgement to Reviewers of Biosensors in 2019
}

\author{
Biosensors Editorial Office
}

MDPI, St. Alban-Anlage 66, 4052 Basel, Switzerland

Published: 16 January 2020

The editorial team greatly appreciates the reviewers who have dedicated their considerable time and expertise to the journal's rigorous editorial process over the past 12 months, regardless of whether the papers are finally published or not. In 2019, a total of 140 papers were published in the journal, with a median time to first decision of 16 days and a median time from submission to publication of 46 days. The editors would like to express their sincere gratitude to the following reviewers for their generous contribution in 2019:

\begin{tabular}{ll} 
Abdallah, Ali & Bohbot, Jonathan \\
Agrawal, Anant & Bonyár, Attila \\
Alfinito, Eleonora & Borile, Giulia \\
Ali, Azahar & Bose, Debojit \\
Ali, Monsur & Bottari, Fabio \\
AL-Naji, Ali & Bykov, Alexander \\
Álvarez, Lorena & Byrne, Hugh \\
Antoce, Arina Oana & Calabretta, Maria Maddalena \\
Armijo, Juan Francisco & Calamanti, Chiara \\
Ashley, Jon & Campos, Rui \\
Atobe, Masakazu & Campuzano, Susana \\
Azoug, Aurélie & Caneira, Catarina R. F. \\
Badea Doni, Mihaela & Cappello, Tiziana \\
Baffa, Oswaldo & Caro, Carlos \\
Bagal-Kestwal, Dipali R. & Carrillo, Jeniffer \\
Bailón, Raquel & Casalini, Stefano \\
Banchelli, Martina & Casteleiro-Roca, Jose Luis \\
Barberis, Antonio & Castro Do Amaral, Gustavo \\
Barbosa, Ana Isabel & Cavallari, Marco Roberto \\
Barfidokht, Abbas & Cellini, Antonio \\
Barrea, Luigi & Chang, Chia-Chen \\
Bartsch, Ronny & Chaturvedi, Prachee \\
BelBruno, Joseph & Chen, Liang-Bi \\
Beni, Valerio & Cheng, Ji-Yen \\
Bertoncello, Paolo & Cheng, Zhiqiang \\
Bhalla, Nikhil & Chiadò, Alessandro \\
Bhattarai, Jay K. & Chiappini, Andrea \\
Blair, Ewen O. & Chiriacò, Maria Serena \\
\hline
\end{tabular}


Cho, Eun Jeong

Colominas, Sergi

Costantini, Francesca

Costa-Rama, Estefanía

Costello, Ben De Lacy

Credi, Caterina

Criado Fernández, Alejandro

Daems, Devin

Dai, Jing

Dang, Vinh

Daunys, Gintautas

Davis, Frank

De Boer, Nanne K

De Marcos Ruiz, Susana

De Souza Gil, Eric

Denoual, Matthieu

Di Nardo, Fabio

Diaz, Sebastian Andres

Dominguez, Rocio B.

Dos Santos, José C.S.

Dosche, Carsten

DS, Francisco Javier

Du, E(Sarah)

Dundon, WIlliam

Ebrahimi, Aida

Ebralidze, Iraklii I.

Esposito, Flavio

Evtugyn, Gennady

Faisal, Shaikh Nayeem

Fateixa, Sara Isabel Augusto

Favero, Gabriele

Fengchun, Tian

Fenton, Brock

Fernandes, Elisabete

Fernández, Antonio

Ferraro, Davide

Fiedler, Goeran

Fitter, Jörg

Foguel, Marcos Vinicius

Fudickar, Sebastian

Fuehrmann, Tobias

Funari, Riccardo

Gamella Carballo, Maria
Gangaraju, Rajashekhar

García, Tania

Gartia, Manas Ranjan

Gaudin, Valérie

Gebicki, Jacek

Gheorghiu, Eugen

Giungato, Pasquale

Goel, Pratibha

Gorodkiewicz, Ewa

Gorski, Waldemar

Goswami, Nirmal

Goto, Takaharu

Griffith, Henry

Grossi, Marco

Güntner, Andreas

Gutiérrez, Juan Manuel

Halder, Avik

Han, Soo Deok

Härmä, Harri

Hassan, Sammer-ul

Havran, Ludek

He, Jiayuan

Henriques, Jorge

Heo, Jinseok

Heveran, Chelsea

Horejs-Hoeck, Jutta

Huang, Chih-Chia

Hung, Chung-Chih

Huotari, Matti

Hwang, Nathaniel

Ignatova, Tetyana

Ionita, Mariana

Iqbal, Hafiz M. N.

Jakieła, Sławomir

Jakubowska, Małgorzata

Jauset, Miriam

Jeerapan, Itthipon

Jemere, Abebaw

Jenkins, Kenneth

Ji, Shaofei

Joensson, Haakan

Jolly, Pawan

Joshi, Tanmaya 
Juan-Colás, José

Kakabakos, Sotirios

Kalogianni, Despina

Kang, Taejoon

Kang, Wenjing

Kant, Krishna

Kassal, Petar

Kaur, Harleen

Kerman, Kagan

Khan, Faheem

Khrustalev, Vladislav

Kim, Jong-hoon

Kitahama, Yasutaka

Kokkinos, Christos

Komarova, Natalia V.

Konijnenburg, Mario

Kraft, Thomas

Krause, Hans-Joachim

Krueger, Eddy

Kujawska, Małgorzata

Kulkarni, Atul

Kumar, Dinesh K

Kumar, Naresh

Kumar, Samir

Kumar, Shailabh

Kusters, Ilja

Lang, Michael

Lee, Gyudo

Lee, Jaewook

Li, Peng

Li, Xiangkai

Li, Zheng

Liew, Oi Wah

Limoges, Benoi ${ }^{\wedge}$

Lin, Abraham

Lin, Hong

Lin, Zuan-Tao

Litti, Lucio

Liu, Weilu

Long, Yuchen

Lou, Shuai

Lu, Yudong

Lukyanov, Pavel
Lyutakov, Oleksiy

Ma, Hanbin

Managò, Stefano

Mandal, Soumyajit

Marasso, Simone Luigi

Marozas, Vaidotas

Marques, M. Matilde

Martini, Elisabetta

Martins, Verónica C.

Mas, Jordi

Massad-Ivanir, Naama

Massera, Ettore

Mehta, Sunali

Melvin, Adam

Memisoglu, Gorkem

Mendez, Francisco

Mendez, Martin Oswaldo

Min, Rui

Minamikawa, Takeo

Minas, Graça

Mishra, Rupesh

Mishra, Rupesh K.

Mitsui, Toshiyuki

Miura, Yoshiko

Miyazato, Hironari

Morasso, Carlo

Moscone, Danila

Moulick, Amitava

$\mathrm{Mu}$, Xuan

Munteanu, Florentina-Daniela

Myllylä, Teemu

Nagles, Edgar

Najdahmadi, Avid

Nallala, Jayakrupakar

Namiesnik, Jacek

Nawarathna, Dharmakeerthi

Nedoma, Jan

Nesmelov, Yuri E.

Neumann, Joachim

$\mathrm{Ni}$, Melody

Nightingale, Adrian

Nikoleli, Georgia-Paraskevi

Nilghaz, Azadeh 
Noort, Danny Van

Novara, Chiara

Núñez Carmona, Estefanía

Nyman, Jeffry

Oćwieja, Magdalena

Orglmeister, Reinhold

Ortis, Alessandro

O'Shaughnessy, Douglas

Osma, Johann

Padalkar, Sonal

Palanisamy, Selvakumar

Palego, Cristiano

Palestra, Giuseppe

Pardy, Tamás

Park, Byung-Wook

Park, Enoch

Park, Min

Pasternak, Grzegorz

Pauliukaite, Rasa

Pedrero, Maria

Pereira, Eulalia

Pérez-Lamela, Concepción

Peter, Van Der Wal

Petrou, Panagiota

Piro, Benoit

Polito, Laura

Polo, Federico

Poltorak, Lukasz

Postma, Marten

Pruneanu, Stela-Maria

Radtke, Aleksandra

Ramsköld, Daniel

Rangnekar, Abhijit

Rao, Ashwin

Rasheed, Tahir

Ray, Partha

Reich, Johannes

Ren, Qinlong

Renken, Christian

Rezaei, Babak

Richardson, Joseph

Robertson, Joseph W.F.

Rodriguez, Natalia M.
Roux, Jean-Maxime

Roy, Dhruvajyoti

Rusdiana, Dadi

Salceda-Delgado, Guillermo

Samiei, Ehsan

Sanchez, Sophie

Sarantopoulou, Evangelia

Scaramuzza, Matteo

Schazmann, Benjamin

Schrittwieser, Stefan

Scida, Karen

Seichepine, Florent

Serafín, Verónica

Šestak, Anamarija

Sgobbi, Lívia Florio

Shahshahani, Amirhossein

Shimizu, Flavio

Shrestha, Namita

Silva, Saimon Moraes

Simm, Roger

Sinyukov, Alexander

Sistla, Srinivas

Somoza, Mark M.

Son, Jongsang

Song, Wuzhou

Sophocleous, Marios

Squella, Juan Arturo

Sriram, Rashmi

Steglich, Patrick

Strömberg, Tomas

Suddala, Krishna Chaitanya

Sun, Meng

Székács, Inna

Szweda, Roza

Taborri, Juri

Tarasov, Andrei

Tavares, Anthony

Teixeira, Sofia Rodrigues

Temiz, Yuksel

Teodorescu, Florina

Ternon, Céline

Tian, Bo

Tischler, Dirk 
Tomasi, Tom

Tonacci, Alessandro

Torres-Ramírez, Eduardo

Travagliati, Marco

Turkyilmaz, Serol

Uberti, Daniela

Ueda, Hiroshi

Ujihara, Masaki

Untersmayr, Eva

Van Dam, Annemieke

Vanegas, Diana C.

Varriale, Antonio

Vazquez, Rebeca Martinez

Vidic, Jasmina

Villarreal-Gómez, Luis Jesús

Vrabec, Tina

Vrentas, Catherine E.

Vullings, Rik

Wagner, Patrick

Walia, Sumeet

Wang, Jianfeng

Wang, Qingqing

Wang, Sai

Wang, Shang

Wang, Yanyan
Weller, Michael G.

Wennmalm, Stefan

Wojciechowski, Adam

Wozniak-Knopp, Gordana

$\mathrm{Xu}$, Jie

$\mathrm{Xu}$, Tingting

Yadav, Marshleen

Yang, Chih-Chin

Yang, Sheng

Yangui, Aymen

Yao, Da-Jeng

Yoon, Sorah

Yu, Feng-Yih

Yu, Jingxian

Zacconi, Flavia

Zakaria, A. B. M.

Zgarian, Roxana Gabriela

Zhang, Jun

Zhang, Lei

Zhang, Ye-Wang

Zhang, Yi

Ziolkowski, Robert

Zolotoukhina, Tatiana

Zwergel, Clemens

(C) 2020 by the author. Licensee MDPI, Basel, Switzerland. This article is an open access article distributed under the terms and conditions of the Creative Commons Attribution (CC BY) license (http://creativecommons.org/licenses/by/4.0/). 